Structuration par genre de l'enchâssement trans-local de l'économie. Exemples d'Afrique de l'Ouest

\title{
Gudrun Lachenmann
}

\section{(2) OpenEdition}

\section{Journals}

Édition électronique

URL : http://journals.openedition.org/apad/228

DOI : 10.4000/apad.228

ISSN : 1950-6929

Éditeur

LIT Verlag

\section{Édition imprimée}

Date de publication : 20 décembre 2000

Référence électronique

Gudrun Lachenmann, « Structuration par genre de l'enchâssement trans-local de l'économie.

Exemples d'Afrique de l'Ouest », Bulletin de l'APAD [En ligne], 20 | 2000, mis en ligne le 24 mars 2006,

consulté le 08 septembre 2020. URL : http://journals.openedition.org/apad/228 ; DOI : https://doi.org/ 10.4000/apad.228

Ce document a été généré automatiquement le 8 septembre 2020

Bulletin de I'APAD 


\title{
Structuration par genre de l'enchâssement trans-local de l'économie. Exemples d'Afrique de l'Ouest ${ }^{1}$
}

\author{
Gudrun Lachenmann
}

Introduction

1 Les recherches sur les femmes et les théories sur le genre disposent, à l'examen, d'un stock intéressant d'études empiriques et d'approches conceptuelles et théoriques. Celles-ci ont contribué à approfondir les fondements des sciences sociales dans la théorie du développement en général. Leur apport a été particulièrement important en sociologie et en anthropologie du changement social. Ces études ont renouvelé les paramètres des analyses du développement appliqués dans la coopération internationale. Les activistes féministes et leurs réseaux internationaux ont réussi à placer le genre sur l'agenda international. Il s'est avéré cependant, d'une part, que les efforts de promotion féminine et aux déterminés par l'approche genre n'ont pu arrêter les effets pervers de développement conduisant à la féminisation de la marginalisation en économie et politique. D'autre part, l'approche "genre" telle qu'elle est intégrée à tous les secteurs et à toutes les politiques de développement n'est pas satisfaisant. La plupart du temps, l'intitulé "genre" couvre encore une approche du rôle des femmes, une approche théorique depuis longtemps critiquée parce qu'elle ne prend pas en compte les relations de pouvoir dans la société, les constructions de genre dans les institutions ni, de façon générale, la structuration par genre de la société.

2 Mon objectif est ici d'appliquer une perspective genre portant sur le caractère systémique et la dynamique des constructions sexuées afin, d'une part, de mieux comprendre certains changements dans la société, et de l'autre, de rendre compte des nouvelles approches théoriques utiles pour la recherche sur le genre. Introduire cette perspective genre serait ce qu'en Anglais on comprend par "engendering". Dans mon article, je propose montrer l'utilité de cette perspective pour l'étude de l'économie 
locale et trans-locale. Dans le contexte des interactions rurales et urbaines en matière de circulation et de mobilisation des ressources, la "complexité et l'intensification des mouvements circulatoires de biens, de symboles, d'idées, et de la mobilité des personnes reconfigurent espaces urbains et ruraux" (Fall \& Manso 2000: 7). Aborder ces reconfigurations sous l'angle des rapports de genre approfondit l'approche proposée et permet cet approfondissement par son caractère constructiviste, relationnel, dynamique dans rapproche des espaces différenciés masculins et féminins, tout en prêtant un intérêt particulier aux interfaces (Long 1992) entre logiques d'action et espaces.

3 J'adopterai cette perspective "genre" en y intégrant des débats récents à propos, d'une part. du concept de trans-localité comprise ici comme la socialisation de l'espace/ temps (Appadurai 1991, 1998), et d'autre part, du concept d'enchâssement ou même de ré-enchâssement de l'économie utilisé récemment dans la nouvelle sociologie économique, dans la ligne des débats anciens de Polanyi (Granovetter, 1985, 1992). Il est sous entendu que l'usage des deux concepts se fait dans le contexte des théories de la mondialisation. Les processus de localisation et de ré-localisation (Long, 2000) en sont constitutifs et l'observation relance, d'un point de vue méthodologique, les intrications du social et de l'économique.

4 Mon hypothèse est que les relations de genre structurent tant la constitution d'espaces trans-locaux (Appadurai, 1991 ; 1998) que l'enchâssement de l'économique en général. En mettant ces rapports en évidence, j'espère surmonter des anciennes et nouvelles dichotomies encore sous-jacentes en sociologie du développement et de la mondialisation. La mise en évidence d'une structuration sociale par le genre pourra faire mieux comprendre les changements des relations villes-campagnes constitutifs des changements sociaux et de l'économie.

5 J'illustrerai ce propos par mes recherches empiriques en Afrique de l'Ouest (Sénégal 1998 ; Cameroun 1996) sur les mouvements paysans, les transformations des relations de genre et des modes d'économie locale "féminine", dans le cadre de la décentralisation et en relation avec des projets de coopération concernant la protection des ressources naturelles. La nécessité de tenir compte de l'enchâssement du local dans les relations trans-locales sera, dans le cadre de ces processus, démontrée à partir de deux exemples de projets de la coopération allemande - l'un au Cameroun, l'autre au Sénégal - tout en regardant la question de participation et de communauté.

D'autres travaux empiriques réalisés à l'université de Bielefeld dans le cadre de travaux de recherche (souvent des thèses de doctorat), et concernant les questions de l'économie locale "féminine", rurale ou urbaine, souvent enchâssée dans des relations trans-locales, viennent à l'appui de mon propos (par exemple Becher 1996, Desai 1999, Laaser 2001, Müller 1999, Nageeb 2000, Padmanahban 2000, Peleikis 1998, Schneider 2000, Schultz 1996, Waibel 1998, Wanzala 2000a, 2000b).

Contributions méthodologiques d'une approche genre

7 Me plaçant dans la perspective des analyses féministes récentes, lesquelles ont démontré que l'économie est structurée par les rapports de genre (Elson 1995) j'espère dépasser la séparation encore très fréquente entre, d'un côté, les analyses en termes d'impact" des changements des structures macro-économiques sur la situation locale et, de l'autre, le champ des recherches sur l'économie locale et ses extensions commerciales jusqu'aux réseaux mondiaux. Pour analyser les activités économiques 
locales et trans-locales enchâssées dans des environnements socioculturels différents, de nouvelles approches méthodologiques sont nécessaires.

Mon objectif, en adoptant une perspective, "genre" à plusieurs niveaux de l'analyse des rapports sociaux, est de montrer que des dichotomies plus théoriques que réelles, pratiquées dans l'analyse des relations intersectorielles, telles que ruraux-urbains, formels-informels, économie de marché-économie de subsistance, explosent sous le choc de l'observation des faits. J'avance donc le propos que la structuration par genre est au cœur de l'effort méthodologique fondamental, à savoir le dépassement d'une tradition épistémologique consistant à penser le social en dichotomies tant en sociologie qu'en Anthropologie sociale.

9 Pour saisir le caractère relationnel de la trame économique j'utilise le concept d'économie féminine (Lachenmann 1996a ; 1997, cfr infra), au sens, non pas d'une unité économique figée, isolée, mais d'une position fluctuante dans des relations, réseaux et niveaux complexes. A ce propos, le concept de modes alternatifs d'accumulation qu'introduisent Geschiere \& Konings (1993) pour élucider la relation entre État et économie semble très utile. Le recours à ces concepts permet d'appréhender les phénomènes tout en dépassant les vieilles unités méthodologiques de ménage et de communauté "traditionnelle", depuis longtemps critiquées en anthropologie sociale (voir p.ex. Guyer 1988) et dans la théorie du développement, en raison de leurs effets uniformisants (Joekes \& Kabeer 1991). L'usage de ces concepts permet de reconfigurer les réalités sociales rurales et urbaines en termes de relations.

Les femmes sont, depuis longtemps, à travers leur insertion dans l'agriculture de subsistance, les porteuses des relations entre villes et campagnes. Qu'entendre ici par agriculture de subsistance? La reproduction et la production centrées sur l'économie et la communauté locales, caractérisées par une logique locale dont les articulations aux systèmes formels des marchés développent des dynamiques nouvelles. Dans le même temps, on constate que la permanence relative des femmes permet de garantir l'accès à la terre dont sont propriétaires des hommes migrants, et même, qu'elles représentent, dans certains nouveaux discours locaux, la continuité ce qui y est désigné par la "communauté traditionnelle" (Lachenmann 1996b; Becher 1996). Pour le commerce, à tous les niveaux, les rapports de genre, avec les différentes formes de coopération qu'ils structurent, sont décisifs.

11 Utilisant le concept d'espace trans-localement construit à partir des relations sociales, on peut montrer que les groupements de femmes et le secteur associatif constituent de plus en plus des réseaux ruraux-urbains. On pourrait donc se demander si les espaces et les débats publics à propos des changements sociaux (Laohenmann 1994), des lieux de parole que génèrent dans une large mesure des mouvements de femmes - ou, comme on le dit de plus en plus, des féminismes - organisés de façon trans-locale et transnationale, jouent une rôle dans la gestation des sociétés.

On peut, par ailleurs, étudier la relation entre l'État et "le local" dans cette perspective, notamment en analysant des événements tels que la "quinzaine de la femme" fêtée, notamment, au Sénégal, ou encore, à travers la mise en scène des organisations locales de femmes à cette occasion. De façon plus générale, les mesures de décentralisation en cours avec l'appui des bailleurs de fond extérieurs peuvent constituer une nouvelle forme de "captation" (Hyden 1980) urbaine des villages : les fonds injectés impliquent des contre parties et portent ainsi atteinte à l'autonomie en matière d'accumulation et de gestion des ressources. 
13 Il devient clair que l'approche selon le genre est indispensable à ce changement de paradigme et que l'on ne peut faire l'économie des rapports de genre dans l'abord de la structuration de différents espaces, logiques etc. Cette approche est. totalement différente d'une analyse du "rôle des femmes" qui, depuis longtemps, a été critiquée en sociologie pour son caractère statique et inapte à percevoir les dynamiques relationnelles et interactives des réalités sociales et de leurs transformations, pas plus que les champs de pouvoir. Cela veut dire que l'intention méthodologique est d'étudier les recompositions sociales et socio-économiques en prenant comme axe les changements dans les rapports de genre, c'est-à-dire la structuration par le genre d'une société. Pour cela, les différentes logiques des acteurs évoluant dans ces configurations, constitueront un point d'entrée de l'analyse qui portera sur les relations à distance les plus typiques.

L'économie féminine enchassée

l'on se situe dans ce cadre conceptuel, l'analyse de l'économie féminine met en lumière les processus relationnels qui font la trame des activités quotidiennes au sein de secteurs, de champs d'activités, et qui forcent à dépasser les séparations toujours vivaces en économie et en politique de développement, entre secteur économique formel et informel, secteur productif et reproductif. Les processus de formalisation ou d'informalisation, en ce qui concerne, par exemple, l'accès à la terre, sont ainsi mis en évidence, de même que les entrecroisement des tâches domestiques reproductives et de la production destinée à la commercialisation. Le maraîchage, introduit partout en Afrique de l'Ouest surtout comme activité génératrice de revenus féminins, en est un bon exemple. Le concept d'économie féminine permet également de mettre en évidence les différentes formes de coopération, et d'échange entre femmes et hommes ou entre différents réseaux de femmes construits, notamment, sur la parenté en ligne maternelle ou sur l'origine locale de l'épouse. Le concept de modes alternatifs d'accumulation (Geschiere \& Konings 1993) contribue à faire appréhender les phénomènes pertinents.

15 Les rapport de genre structurent, certes, ces domaines, mais l'intérêt d'une approche en termes de dynamiques relationnelles dépasse ces rapports et peut s'appliquer à différents niveaux de l'économie et des institutions sociales. La complémentarité entre des ressources tels que la force de travail ou le savoir, les rapports productifs marqués par le genre, qu'il s'agisse de l'unité de production domestique où d'une exploitation orientée vers le marché, l'interaction entre secteur reproductif et productif, la structuration des marchés et des activités économiques, les interactions entre secteur formel et informel sont autant de domaines d'application de cette approche.

En analysant les rapports de genre on est automatiquement amené à restituer le lien, qui existe dans la réalité, entre subsistance et marché, secteur productif et reproductif. Sous l'impact d'une diversification économique, les échanges et formes de coopération entre hommes et femmes prennent, eux aussi, des formes de plus et plus variées. Souvent, les circuits des ressources (monétaires, travail, équipement, intrants etc.) pour les différentes activités agricoles saisonnières passent par sur l'accès différencié à ces ressources suivant le genre (Becher 1996). Les rapports de genre modulent l'accumulation du capital nécessaire à un début d'entreprise, que ce soit à travers les tontines et les contributions qui y sont faites, ou à travers les conditions d'exclusivité de genre mises à l'octroi de crédits par certains programmes de développement. A un niveau plus élaboré l'analyse des champs d'activité économique des femmes urbaines 
montre la complexité de l'intrication des rapports de genre tout au long du continuum qui lie la reproduction et le commerce transnational. Ainsi l'enchâssement des activités économiques dans les relations sociales est constitutif de la nouvelle articulation des sociétés autour des relations villes-campagnes.

Tout en dépassant les anciens concepts de ménage et de communauté et en tenant compte des reconfigurations relationnelles des liens sociaux entre villes et campagnes, on peut montrer que les relations entre ménages, au-delà des unités de production, suivent dans une mesure importante et souvent invisible dans une analyse classique, les liens entre femmes, qu'il s'agisse d'échange de ressources, de savoir et de techniques, ou d'entraide et de sécurité sociale.

Le concept de ménage doit aussi être appréhendé autrement qu'il ne l'est: l'unité domestique n'agit, en effet, pas toujours avec une rationalité commune, et n'est pas non plus un lieu obligé de conflit ou de coopération (Sen 1981 ; Joekes \& Kabeer 1991). La réalité est plus nuancée. En ce qui concerne les rapports entre femmes, il peut se faire que, par exemple, des co-épouses coopèrent de manière trans-locale, recourant à des ressources économiques et sociales dont toutes tirent profit. Il arrive aussi de plus en plus que la capacité de trans-localisation sociale repose sur l'implantation des logements et des lieux d'activités économiques de deux (ou plusieurs) épouses. Dans la plupart de cas, l'une d'elles vit en ville et l'autre "en brousse" ; un commerce s'organise sur la base d'une complémentarité des opportunités. Bien sûr, il se pose toujours le problème de la monétarisation de ces relations, les femmes actives dans l'économie rurale pouvant craindre que le produit commun, peut-être vendu par le mari sur le marché, soit consommé en ville, par une autre femme.

Le mari, avec ses différents "bureaux", est un intermédiaire dans cette relation entre des économies urbaine et rurale, des secteurs formel et informel. Les femmes représentent les différents secteurs économiques, mais également les espaces "modernes" ou "traditionnels" où se complètent une femme "intellectuelle" et une femme "traditionnelle" -suivant les stéréotypes que beaucoup utilisent, tant en ville qu'à la campagne. Je pense à l'exemple d'une femme du Sine Saloum (Sénégal) qui a pu poursuivre ses études, puis des activités professionnelles, grâce à sa coépouse, non formellement instruite, et qui prend en charge l'ensemble du ménage, enfants de la coépouse compris. Les modes d'accumulation spécifiques font que les femmes intellectuelles peuvent incarner le lien à l'État, autant que le secteur informel ou le commerce. En même temps, leur activité "moderne" n'est possible que grâce aux "petites bonnes" immigrées de la campagne.

Les configurations varient selon les responsabilités monétaires et les parts d'investissement en capital, (Frey-Nakonz 1984). Souvent la mère, grâce à ses activités économiques propres, cède un capital de départ à sa fille. Si le mari fait cet investissement, il se sentira libéré d'autres obligations, et ne prendra plus en charge les frais scolaires des enfants, par exemple. Les femmes s'insèrent le plus souvent dans une "logique de subsistance", c'est-à-dire qu'elles assurent surtout les bases de l'existence (livelihood) y compris l'énergie domestique, la consommation d'eau etc., ce qui oriente aussi leur manière de s'occuper des ressources naturelles. Dans leur économie féminine, elles établissent donc de manière privilégiée le lien entre économie rurale et urbaine en ce qui concerne les questions de subsistance. Par contre, si les hommes font le lien, ils investissent en général leurs ressources "urbaines" (pensions etc.) dans des cultures de rente au sens classique. Toujours est-il qu'une grande partie des revenus 
passe en boissons et ailleurs, en d'autres dépenses ostentatoires - y compris souvent des fêtes des urbains dans leur village d'origine à l'occasion d'enterrements ou pour des vêtements et bijoux d'eux mêmes et de leurs femmes urbaines - comme j'ai pu constater au Cameroun et au Congo (au temps où il s'appelait Zaïre).

21 Les femmes, récemment qualifiées de "community managers" dans les milieux de la coopération internationale, gèrent les ressources naturelles au niveau de l'économie familiale et au sein de la communauté. Dans une approche relationnelle il faut donc voir les relations de coopération, d'accès et d'entretien dans un cadre plus large. Les femmes ont, cela tout le monde en est conscient, besoin de temps pour entretenir les ressources naturelles, et il faudra donc alléger leurs travaux. Mais la question est de savoir comment les moyens d'alléger leurs travaux seront financés. Les hommes fourniront-ils un apport monétaire pour l'investissement et le maintien en activité d'un moulin céréalier? Si ce capital ne provient pas des cultures de rente, proviendra-t-il de la migration vers les villes? Par ailleurs, les femmes urbaines, intellectuelles, commerçantes etc. contribuent souvent, à l'aide de leurs ressources monétaires propres, aux obligations des maris à J'égard de leur famille d'origine, tout en assurant l'accès aux ressources rurales telles que le foncier ou l'équipement technologique (Schneider 2000).

22 En considérant ce qui a été mentionné plus haut à propos de certains échanges et apports, soit pour assurer la production rurale, soit pour appuyer les dépenses typiques en villes pour services, éducation etc., il me semble que les rapports de genre sont en général les plus monétarisés des rapports sociaux entre ville et campagnes. Les femmes rurales sont souvent très dépendantes des transferts monétaires en raison des besoins monétaires croissants nécessaires pour pouvoir subvenir aux besoins quotidiens de leur famille, mais aussi pour satisfaire toutes sortes d'exigences des associations et groupements locaux tels que les tontines: il faut cotiser. Les politiques d'ajustement structurel font que tous les dispositifs de développement exigent le recouvrement des coûts des services de santé ou des investissements ruraux en matière d'approvisionnement en eau ou d'autres infrastructures. Cette monétarisation des relations sociales, y compris celle des prestations de travail entre parents, des soins aux personnes âgées, de l'accès à la terre etc., a des conséquences évidentes pour la reconfiguration de la société, aboutissant parfois à des phénomènes de "vénalité" (Elwert 1987).

23 De leur côté, les femmes urbaines très mobiles et inventives, peuvent disposer plus facilement de numéraire. Leur apport monétaire au monde rural déterminera, dans une large mesure, le maintien de leur appartenance au milieu d'origine. Par exemple, elles sont souvent obligées de payer leur accès à la main d'œuvre ou à la terre. C'est aussi par des dons d'argent qu'elles pourront se libérer des obligations sociales, telle que des prestations en travail pour leur belle-famille, comme c'est le cas, notamment, au Zimbabwe (Schneider 2000). De façon générale, J'accès des femmes à la terre est plus liée à la monétarisation, et contribue ainsi, à son tour, à une monétarisation croissante des campagnes, voire à la vénalité des responsables, lorsque les cadeaux et la corruption ouvrent la voie de l'achat de terre. On pourrait citer, de ceci, de nombreux exemples. Au Nord du Ghana (Becher 1996) des parcelles dans un périmètre irrigué sont attribuées pour une année. Ceci paraît ne pas devoir bouleverser de manière permanente le mode d'accès traditionnel à la terre basé sur un certain ordre de genre (à savoir que c'est par leur mariage au sein d'un groupe déterminé que les femmes ont 
normalement accès à la terre) les femmes ont, en majorité accès à ces parcelles. Elles peuvent acheter les intrants nécessaires grâce aux revenus des (petites) activités économiques constituant une extension de leurs travaux de subsistance: vente de certains fruits, de produits transformés ou de plats cuisinés. Toutefois, elles ne pourront garantir leurs droits de propriété que si elles sont riches, de bonne famille et en possession de forces magiques (cas du Cameroun, notamment).

Dans les migrations, les interactions rurales - urbaines passent, depuis longtemps, par les femmes qui se maintiennent dans l'agriculture tout en assurant "l'identité rurale et traditionnelle" de leur groupe social. Il faut cependant discerner de nouvelles formes d'interactions, par exemple entre femmes 'intellectuelles' en ville et femmes dans les zones rurales. Dans des situations précaires telles qu'une sécheresse, les femmes s'assurent des points d'ancrage en ville et, en accumulant les gains qu'elles y font dans le petit commerce, maintiennent leurs attaches rurales, par exemple dans le cas des sociétés nomades du Kenya ou du Niger, à travers la restitution de cheptel à leur famille d'origine (Schultz 1996; Waibel 1998). Dans certaines régions, les migrations urbaines, souvent saisonnières, des jeunes filles mettent sérieusement en question l'identité de la "société traditionnelle", par leur conduite considérée comme immorale, au grand dam des responsables de mouvements paysans. C'est le cas, notamment, du Sine Saloum très islamisé.

Différentes formes de coopération structurées par des rapports de genre sont décisives pour le commerce. Au niveau intercontinental, les commerçantes ghanéennes (Amponsem 1996), utilisent des réseaux féminins pour s'informer sur les routes, les opportunités, les fournisseurs, les voyages en communs, les réseaux de clientes, les possibilités d'hébergement etc... Elles s'organisent en utilisant ponctuellement la coopération des hommes, souvent des migrants, y compris ceux qui travaillent dans le secteur formel. Je mentionne, par exemple, le cas d'une commerçante du marché de Kumasi, importatrice de pièces de voitures d'occasion, grâce à la collaboration de son neveu employé dans un atelier en Allemagne. En Asie, à Bangkok, des migrants masculins offrent leur assistance pour les formalités de transport et de financement des achats : la commerçante investit en marchandise l'argent que les migrants souhaitent faire parvenir à leur famille et remet la somme à la famille après vente de la marchandise, dans ce cas des objets en matière plastique. Parfois, à l'inverse, ce sont des épouses émigrées qui constituent les bases ou les succursales de commerces trans-frontaliers. Le contact avec les fournisseurs et les clients passe souvent par les femmes qui opèrent selon des modalités qui leur sont propres. Les "modes alternatifs d'accumulation" entre secteur formel (État ou privé) et informel, tels qu'ils ont été élaborés par Geschiere/Konings (1993), s'appuient essentiellement sur des rapports de genre. A Nairobi au Kenya, par exemple, les "business women" ont toutes été directement ou indirectement intégrées dans les services publics, qu'elles y aient travaillé elles-mêmes ou qu'elles y aient leurs contacts à travers leur mari. Elles peuvent utiliser les facilités et les avantages liés à ces fonctions : accès à l'information, au crédit, à l'immobilier, et bien sûr, elles bénéficient d'une protection contre l'État prédateur, échappant aux lourdes taxations informelles de la corruption (Laaser 2001).

Dans le domaine du commerce des produits vivriers, la situation, très complexe, est en transformation dans les villes. Dans le secteur informel, les femmes essaient de plus en plus de dominer le marché des plats préparés. Au niveau formel, par contre, la privatisation des offices de commercialisation, semble faire évoluer le commerce au 
détriment des femmes, les coopératives masculines étant surtout encouragées. Un processus analogue s'observe quand la transformation s'industrialise, et cette industrialisation est hautement recommandée par la politique du développement ; c'est notamment le cas au Cameroun.

Les analyses des différents champs d'activité économique des femmes urbaines (voir notamment, pour Harare, Zimbabwe, Schneider 2000) montrent la complexité des intrications dans un continuum entre reproduction et commerce international (par exemple, produits artisanaux divers faits à la main et commercialisés à travers le continent), un continuum établi sur les rapports villes-campagnes où les obligations sociales à l'égard des familles des beaux-parents ont leur place. Cet exemple montre le caractère trans-local de la production domestique de subsistance, utilisée pour la vente dans la cour ou à la rue : les matières premières et la main-d'œuvre proviennent des réseaux familiaux ruraux-urbains. La production provenant de groupements et de formations en apparence peu progressistes, tels que les clubs de femmes chrétiennes et les foyers sociaux (articles de tricot et crochet) est vendue par une voisine insérée dans le secteur formel local, ou à travers les réseaux organisés sur la longue distance, Afrique du Sud incluse. Ici, comme dans le commerce à très longue distance et à plusieurs paliers sociaux, des relations personnelles entre femmes et migrant(e)s sont fondamentales, mais ces relations sont entretenues et utilisées par une personne de confiance. Cette personne reçoit des commandes provenant de réseaux entiers: commandes d'achat de matériaux et de pièces ainsi que de produits de consommation déterminés. Ces réseaux s'appuient d'une certaine manière sur des rapports de genre, les femmes voyageant, par exemple, avec certains chauffeurs qui leur assurent une protection. Dans le cas susmentionné des Ghanéennes (Amponsem 1996) voyageant jusqu'à Bangkok, la commerçante combine l'achat de pièces détachées d'occasion par des neveux émigrés en Allemagne et la fourniture de produits alimentaires à des magasins africains en Allemagne des magasins gérés par des hommes migrants de provenances africaines diverses et destinés à une clientèle africaine migrante. Suivant les recherches de Bazenguissa-Ganga (2000) des femmes originaires du Congo Brazzaville entretiennent des nganda et créent un style de vie propre basé sur une consommation très chère, dans des bars informels ou illégaux où les hommes actifs dans des activités lucratives illégales, comme le trafic de drogue, trouvent une atmosphère particulière.

Le secteur associatif comme acteur de l'espace trans-local

Le concept d'espace construit trans-localement, à partir des relations sociales des femmes, nous permet de montrer que les groupements de femmes constituent de plus en plus des réseaux ruraux - urbains. Souvent les relations ont été analysées comme perpétuant les relations dominantes urbaines, avec les membres des mouvements féminins, ainsi que les femmes socio-technocrates oppressant les femmes de la base. Mais cela change, et l'on observe ce qu'on appelle en Amérique Latine des "féminismes populaires". Tout en dépassant ces clivages, on peut dire que les mouvements trans-locaux de femmes constituent un élément structurant important des sociétés civiles. Je pense aux réseaux qui constituent des espaces de négociation de nouveaux concepts de développement, mais aussi aux contacts féminins familiaux transportant des idées islamistes, comme c'est le cas entre femmes urbaines libanaises migrées en Cote d'Ivoire, Abidjan, et femmes dans des villages d'origine au Liban (Peleikis 1998). 
29 Dans le monde du "développement", les espaces sociaux reconnus se sont, eux aussi, quelque peu délocalisés. Certes, des lieux de conférence, le plus souvent dans les grands hôtels, sont encore recherchés comme localisation urbaine par certains groupes de femmes. Dans l'ensemble, ces espaces sociaux s'identifient plutôt à une société rurale/ urbaine unissant des femmes d'un niveau d'éducation moyen et orientées vers la pratique et constituent une identité particulière fondée sur les rapports entre villes et campagnes. Les femmes concernées sont apparentées ou alliées à des responsables politiques ou à d'autres familles influentes dans le monde urbain, souvent à travers leur mari. J'ai soulevé ailleurs la thèse (Lachenmann 1996a) que, dans une large mesure, la légitimation politique des mouvements paysans est assurée de cette manière, ce qui peut leur faire perdre leur autonomie tout en cherchant des "complices" dans la politique politicienne (Sénégal). On voit aussi de ces "leaders femmes", très conformes aux modèles dominants, et engagées dans des courants de type "développement communautaire", soucieuses d'"éduquer les femmes". On risque alors de voir, à chaque fois, les mêmes voyageuses se déplaçant de réunion de bailleurs de fonds en réunion de bailleurs dé fonds. Les ONG sont championnes en la matière.

30 Des types de femmes assez autonomes émergent pourtant dans le cadre des mouvements. Il s'agit, le plus souvent, de femmes qui combinent leur responsabilité de "leader" avec des qualifications et des activités économiques et commerciales. Quelquefois, cependant, les femmes qui réussissent à élargir leurs affaires dans des sphères d'activité classiques, telle que les buy'em-sell'em au Cameroun, sont considérées comme exploitant les femmes rurales, tandis que des hommes d'affaire nouveaux entrent dans le système et achètent sans intermédiaire la récolte des groupements de femmes. Dans le cas rapporté par une étudiante Camerounaise (communication orale, Friede Ngo Youmba), l'ancienne bu'yem-sell'em, qui assurait le lien entre les productrices et le marché urbain la commerçante ne peut rien faire dans cette situation. Elle perd les investissements qu'elle a faits pour financer la production des femmes paysannes et craint d'affronter les accusations de sorcellerie qui ne manqueraient pas d'accompagner son succès économique dans le système urbain moderne (Fisiy \& Geschiere 1993).

31 Au Sénégal, j'ai observé que le processus d'ascension des femmes, dans le monde politique, mais aussi dans le monde associatif, s'arrête à un certain niveau. Les femmes sont ensuite incitées à rentrer dans le rang par le biais d'une intégration dans la politique nationale, en particulier la politique de "promotion féminine". Le mouvement paysan compte, cependant, des femmes influentes. De retour dans un village appartenant à une communauté rurale de la région de Kaolack, nous avons pu constater que le président de l'organisation paysanne nous a salué mais guidé ensuite chez la vice-présidente qui nous a logées. Elle dispose d'une certaine liberté personnelle et est relativement aisée étant donné qu'elle fait du commerce. Elle fait accepter son succès : elle donne plus qu'elle ne gagne, dit-elle. Le président a plusieurs jeunes femmes et est très actif dans le commerce céréalier local. On peut donc dire qu'il y a une re-traditionalisation des hommes du mouvement paysan, mais aussi, une urbanisation de certaines femmes. Les groupements sont toujours surtout constitués par des femmes, bien qu'après la récente répartition de céréales des Banques Céréalières des candidatures d'admission de membres masculins se présentent à nouveau (Blundo 1995). 

D'autre part, la relation entre le monde urbain et le monde rural s'intensifie à travers leurs réseaux. Au Cameroun, à Bamenda (Lachenmann 1992), des femmes urbaines, actives dans des groupements très conservateurs de l'église presbytérienne, c'est à dire des institutrices, des employées d'entreprises agro-économiques etc., gèrent des débats sur les questions de la violence mais aussi de la résistance contre la répression du régime politique. Au Sénégal, moyennant, il est vrai, un support extérieur, on voit réaliser des publications et des études sur la situation juridique, et s'organiser des débats avec des membres féminins du parlement, des journalistes, voire même des syndicats (Diop 1995). Elles influencent certainement les initiatives en matière de droit de l'homme, de lutte contre le VIH, d'initiatives contre la violence, etc.

Outre les groupements de promotion féminine et leur Fédération (membre du Conseil National de Concertation des Ruraux), il y a, au Sénégal, une autre organisation de niveau national, la Fédération des Associations Féminines du Sénégal (FAFS). Celle-ci a installé des bureaux régionaux pour lesquels elle recherche des monitrices, et en général, elle essaie d'obtenir des financements extérieurs pour fonctionner comme agence d'appui à l'auto-promotion. Aussi, une organisation centrale a été fondée, le Conseil Sénégalais des Femmes COSEF, qui édite un Bulletin de Liaison "COSEF Info" (auprès de l'Institut Africain de la Démocratie), et dont les membres sont des femmes individuelles ainsi que des groupements. Les activistes semblent cumuler certains fonctions, soit à titre de volontariat, soit comme consultantes locales avec certains financements, par exemple pour une programmation régionale de projets de lutte contre la pauvreté. Ces cumuls semblent permettre de s'intégrer dans des cercles de développeurs, c'est-à-dire de devenir courtiers du développement (Bierschenk, Chauveau, Olivier de Sardan 2000), et, dans ce rôle, de donner un certain pouvoir de conceptualisation et d'influence auprès des mouvements paysans. Ils donnent la possibilité d'assister à des ateliers, de prendre part à des voyages organisés surtout par des ONG étrangères. Les quelques femmes remplissant des fonctions relativement importantes, telle que présidente d'association, si elles contribuent à une certaine légitimation politique des associations, ne gagnent pas en pouvoir: leur activité est rapidement captée par l'État.

Concertation des Ruraux, fondé en 1993) les questions de volontariat et de professionnalisation se posent, en général, pour les hommes. Tout un discours est produit sur le caractère non-démocratique et clientéliste des réseaux intermédiaires du secteur associatif. Le local et le national sont opposés dans ce discours, sans considération pour les réalités de la nécessaire structuration en réseaux. Les problèmes de structuration du mouvement paysan dans sa totalité nationale existent et l'un d'eux est que la base ne perçoit souvent plus la nécessité, ou la possibilité, de contribuer, par ses cotisations aux structures d'organisation au delà du niveau de zone. Par contre, il est clair que la présence publique des leaders actifs dans le CNCR est reconnue par tous les membres, même si l'on peut objecter que l'admiration pour des leaders charismatiques est avant tout un signe d'impuissance dans la société civile.

Notre étude de 1998 (Lachenmann \& al. 1999), qui se voulait une analyse, à travers des études de cas de groupements, de la contribution des mouvements paysans à la constitution de la société civile dans le contexte de la décentralisation, a été réalisée en période de soudure. La situation alimentaire était très précaire dans quelques zones et

Bulletin de I'APAD, 20 | 2000 
villages, et l'inquiétude régnait à cause du retard des pluies. Les mesures adéquates pour remédier à cette situation de famine dans les villages n'avaient pas été prises à temps et la société ne se permettait pas de renvoyer l'État à ses responsabilités. D'un côté, en ville, y compris dans la presse, on a pratiquement pas parlé de la soudure. Par contre, le secteur associatif a organisé un événement spectaculaire en mettant en scène le monde rural. Les leaders des mouvements des ruraux, à travers le CNCR, ont sensibilisé le public en dernière minute, lors d'un entretien télévisé et à travers la presse.

Il était intéressant d'observer à cette occasion le type de mise en scène et de légitimation à travers l'affirmation d'une identité rurale. Les leaders, dont l'un très charismatique, se sont présentés avec bonnet (une pièce vestimentaire typique des ruraux et que, quant à eux, ils ne portent jamais), et en compagnie d'hommes relativement jeunes faisant figure de spécialistes, non pas académiques, mais techniques et issus de régions rurales. Il se trouvait parmi eux, bien sûr, une seule femme, elle aussi présentée comme femme spécialisée moderne. Dans le temps, il y avait même eu des essais de formation d'un Parti Paysan. Dans le contexte de la décentralisation, par contre, les leaders paysans, élus comme conseillers, prétendent représenter le "développement" considéré, semble-t-il, comme équivalant complètement au développement rural. Cependant, les actions prises par le Gouvernement ne furent pas très efficaces. Nous avons assisté à la distribution de riz par le sous-préfet d'une Communauté Rurale : une quantité minime par personne. A partir de ceci, il devient clair que les conceptions gouvernementales en matière de politique alimentaire et de besoins ne coïncident pas du tout avec celles du secteur associatif paysan. La "communauté des leaders paysans" ne semble pas comprendre le sens de la sécurité alimentaire au moment où il existe, sur les marchés, du riz en quantité suffisante à prix subventionné. Ces leaders pensent que l'État et les institutions urbaines "mangent" les aides venues de l'extérieur et destinées aux ruraux. Cependant, il semble que dans quelques villages la situation étaient telle que les populations n'avaient plus de moyens monétaires.

Il est connu, mais rarement pris en considération, que la sécurité alimentaire dont la crise en œuvre se fait entre ville et campagne, est structurée de manière décisive par les rapports de genre. Aux alentours d'une ville secondaire moyenne de la région de Kaolack, - voyait des femmes cherchant quelques dons auprès de l'Église catholique, tandis que les hommes étaient partis dans l'espoir de gagner quelque argent. Il pourrait sembler étonnant que cette situation alimentaire précaire - si ce n'est dans la mise scène de famine décrite ci-dessus - n'entre pas dans le discours politique de l'État, pas plus que dans le discours développementaliste actuel, celui des ONG nationales et des bailleurs de fonds de tout genre inclus. C'est que, officiellement, dans la catégorisation internationale, le Sénégal n'est actuellement pas éligible pour l'aide alimentaire.

La politique agricole tente, de façon bien compréhensible, de promouvoir le marché national des produits alimentaires, donc d'inciter les ruraux à s'occuper d'alimenter les villes plutôt que de se consacrer aux produits de rente pour l'exportation. La structuration de cette production et du commerce par genre est décisive. Dans quelques cas, les hommes pères de famille ont du mal à cultiver des produits vivriers pour la vente, étant donné leur responsabilités de redistribution familiale. Par contre, et contrairement aux accusations d'irrationalité des femmes auxquelles il est reproché d'investir dans les réseaux de voisinage plutôt que dans les systèmes d'épargne-crédit 
des organisations paysannes, les femmes assurent avant tout l'alimentation locale, vendant toujours de petites quantités de céréales obtenues dans les relations de troc avec des commerçants villageois, en vue d'obtenir des produits de première nécessité tels que de l'huile.

Les marchés locaux sont de plus en plus desservis par des leaders paysans achetant les petites quantités offertes par les femmes pour les vendre aux grossistes de passage sur les marchés. Cela est le résultat contre-productif de beaucoup d'efforts pour mettre en place des Banques Céréalières gérées par les associations villageoises afin d'augmenter la sécurité alimentaire dans les villages tout en stabilisant les prix des céréales que les paysans doivent, autrement, ( $r$ )acheter au moment de la soudure si les récoltes d'arachides n'ont pas été suffisantes. Les essais de commercialisation producteurs consommateurs urbains par les mouvements sociaux paysans et urbains n'ont pas donné les résultats attendus. Deux erreurs, au moins sont la cause de cet échec. D'une part, en effet, les mouvements ont compté sur leur travail volontaire sans vraiment calculer les frais de transport, de stockage, etc., et de l'autre, les paysans ont demandé des prix fixes et des avances à leurs propres coopératives de vente, comme ils avaient l'habitude de le faire avec les commerçants et avec l'État.

Décentralisation : état urbain / société rurale captée

La "captation" de la société rurale par l'État (Hyden 1980) à travers les lignes du mouvement associatif, apparaît à travers l'analyse d'événements tels que la "quinzaine de la femme" ou à travers celle de l'organisation de la mise en scène des organisations de femmes. De même, les processus de décentralisation risquent de constituer une nouvelle forme de captation des campagnes par les villes. L'observation participante de la "quinzaine de la femme" en Mars 1998 à Dakar et dans des villes régionales au Sénégal a montré comment les femmes venant de toutes les régions rurales accomplissent, à cette occasion, leur rôle traditionnel en louant le président (ce qui ne l'a pas empêché de perdre les élections en 2000). Depuis pas mal de temps, la légitimation du pouvoir en région rurale passe par la promotion féminine. Dans les villes secondaires, les campagnes de promotion féminine de l'État urbain et les leaders femmes du secteur associatif font passer le message de régionalisation et de décentralisation de manière assez autoritaire.

En ce qui concerne les activités de la Communauté Rurale dans le cadre de la décentralisation au Sénégal, au sein du Conseil de Communauté rurale étudiée dans la région de Kaolack, la secrétaire de la sous-préfecture est la seule femme venant du secteur formel. Son mari, ancien secrétaire en retraite, est responsable des affaires de la $\mathrm{CR}$ et une grande partie des conseillers ne sont pas alphabétisés en français ou, bien sûr, il leur manque le savoir administratif. Ce savoir, malgré les formations en cours (projet "alphabétisation" de la Coopération Sénégalo-Allemande), est bien sûr encore monopolisé par l'État.

42 Les quatre femmes membres de la $\mathrm{CR}$ en question, dont l'ancienne présidente de la $\mathrm{CR}$ même, se plaignent de leur impuissance face à la grande majorité des hommes membres. Elles déplorent surtout de ne pas être membres des commissions importantes, telles qu'Environnement ou Foncier. Elles constatent, de façon remarquable, que les hommes ne "passent pas le message". Cela veut dire que les CR seraient plutôt vues comme des arènes d'information et d'accès à des ressources publiques que de lieu d'influence de la société civile. 
Ainsi, la relation entre ville et campagne est conçue par les villageois comme étant surtout une question de savoir, d'information sur les possibilités offertes par la ville et par les relations modernes de coopération internationale. Dans le cadre des associations paysannes, par leurs leaders jouant les courtiers, les villageois deviennent de plus en plus aptes à capter ces messages. Les rapports de genre y jouent un rôle important, et pas seulement parce qu'il est connu que les bailleurs de fonds y insistent. L'hypothèse peut être lancée que l'État renforce actuellement son emprise sur la population et augmente la légitimité du régime en place à travers la promotion féminine et ceci, précisément, au même moment où la décentralisation produit une certaine uniformisation du mouvement féminin, un processus contraire au principe d'un pluralisme inhérent à l'idée de société civile. Dernièrement, en effet, le Gouvernement a donné aux femmes de soi-disant "Cases Foyers". L'architecture en est très créatrice, d'un style traditionnel modernisé, par contre à l'intérieur il y a surtout une grande salle de réunions et de manifestations, et quelques petites salles ou seront installées, dit-on, des machines à coudre, le tout n'étant pas encore fonctionnel. Simultanément, un programme de sécurité alimentaire pour les femmes aurait été lancé, avec contribution de bailleurs de fonds, prévoyant notamment une Banque Céréalière. Comme le crédit serait arrivé en pleine période de soudure, il fut décidé d'accorder des crédits aux différents groupements féminins. Dans une ville secondaire de la région de Kaolack, ce crédit consistait en une certaine somme à restituer après six mois (février - septembre) avec presque un tiers d'intérêt, un taux que les femmes ne trouvaient même pas abusif. Le programme était administré par des monitrices de l'État, responsables des groupements féminins, ainsi que des comités de gestion respectifs. Il s'agit d'activités telles que le petit commerce où le nombre d'actifs s'accroît, entraînant une diminution des revenus, et non pas d'agriculture ou de jardinage. Il est frappant de constater la persistance d'une logique de partage à l'arrière plan de ces initiatives : les femmes s'attendent toujours à ce qu'on "partage" toutes les ressources monétaires venues de l'État ou de l'extérieur, et non à ce qu'on les investissent conformément à une logique urbaine de business.

La coopération sénégalo-allemande réalise un programme d'alphabétisation de femmes dans certaines régions. Mais autant dans le Projet d'alphabétisation des Conseillers, que dans les activités de développement organisationnel du Projet PAGERNA (cfr infra), le nombre de femmes informées de manière approfondie sur les questions de décentralisation et régionalisation est très faible. Ainsi doit-on craindre que les femmes, si actives dans les groupements de base, ne puissent maintenir leur influence dans les transformations en cours dès que le pouvoir local se trouve institutionnalisé et qu'elles perdent des lieux de débats publics, éléments de société civile.

La décentralisation semble, en effet, mener actuellement à des insécurités, à des contradictions, mais aussi à des opportunités de transformation. On y entrevoit des moyens de contourner les modes autoritaires de gouvernance (Mbembe 1988) et de suppléer aux déficits d'articulation entre les différents niveaux administratifs et politiques. En politique de développement on se réfère à une cohérence verticale, preuve d'un début de prise en compte des structurations sociales et des interactions, des interfaces (Long 1992) entre différentes logiques d'action et de négociation de sens. La question centrale est de savoir s'il s'agit de ne pas créer des nouvelles formes autoritaires mais, au contraire, de rendre possible la négociation transparente entre 
différents intérêts et la recherche de concepts et de modes de gestion des ressources naturelles plus souples et flexibles.

Les approches participatives suivies ces dernières années par les dispositifs de développement risquent de perpétuer la perception dichotomique des ville et des campagnes. Cette approche populiste utilise une conceptualisation simple en termes de systèmes opposés et uniformes et se réfère à des abstractions généralisantes, telles que village ou communauté. Selon moi, les différences internes doivent toujours être regardées en même temps que les relations et interactions, ou encore les capacités de négociation entre différents groupes constitutifs ou avec l'extérieur.

Ce sont ces problèmes, soulevés par ces approches de décentralisation et d'auto-promotion sans considération du contexte social et politique, qui ont suscité l'usage du concept de 'cohérence verticale', évoquant l'articulation des différents niveaux et logiques de gestion. Un autre cas est celui de l'approche "foresterie sociale" introduite dernièrement, sous l'influence des bailleurs de fonds, dans beaucoup de Codes Forestiers. Par sa logique, cette approche prévoit l'autogestion des ressources forestières par la population, à l'inverse des classifications de forêts et domaines d'État, forêts classées et autres réserves naturelles. Cependant, dans la plupart des cas, les pratiques autoritaires ne changent pas encore. Ici, contrairement aux réalités sociales trans-locales, les approches participatives construisent une localité idéalisée à laquelle s'applique une gestion locale. Ces approches débouchent nécessairement sur des impasses économiques et politiques par faute d'une prise en considération de modes institutionnalisés d'interaction avec les niveaux supérieurs.

Les rapports de genre son transversaux à ces relations. Souvent, l'accès à la terre et aux ressources naturelles passe par les relations d'alliance et de mariage. Les femmes ont ainsi parfois accès à des marigots a l'extérieur de leur village de résidence, ce qui est oublié dans les projets d'aménagements de protection des ressources naturelles. Les femmes ne figurent pas dans la "communauté" reconstruite ou réinventée comme traditionnelle. Les nouvelles formes de participation introduites par l'État avec l'appui ou sous la pression de la communauté internationale, ne tiennent souvent pas compte de l'ancienne structure parallèle de représentation des femmes dans les systèmes locaux de pouvoir. Elles ignorent les mécanismes de liaison entre le monde des femmes et les structures générales de pouvoir, c'est-à-dire qu'elles méconnaissent les interfaces entre les femmes et les structures dominantes.

Deux exemples d'institutions introduites dans le cadre de la coopération

e présenterai ici deux exemples d'institutions et d'organisations locales introduites ou renforcées dans le contexte de la coopération au développement :

- foresterie sociale par des "communautés" locales au Sud-ouest du Cameroun (Mount Cameroon)

- cellules d'animation et de coordination CAC incités par un Projet visant à une meilleure gestion des ressources naturelles basée sur l'auto-promotion au Sénégal (Région de Kaolack)

Dans le cas du Cameroun que j'ai eu à étudier brièvement dans le cadre d'une étude sur l'approche genre dans la coopération (Lachenmann 1996b) j'ai pu constater que le concept de communauté mène à une réinvention de la communauté "autochtone" et "traditionnelle". Les relations ville - campagne sont perçues et symbolisées par l'essai d'exclusion des "étrangers" par les "autochtones" et cette perception est le produit d'une structuration de la réalité à l'aide de concepts introduits par le Projet de la Coopération Germano-Camerounaise (Mount Cameroon). Les "étrangers" qui se sont 
installés dans la région depuis plusieurs décennies sont considérés comme profitant trop de l'exploitation des ressources naturelles. Les sociétés d'exploitation de bois en général engagent des journaliers recrutés ailleurs et qui semblent récolter les prunus africanus (destinés à un usage industriel) de manière très destructive. Les locaux ne sont pas demandeurs d'emploi dans ce contexte.

51 Ces relations rurales-urbaines, à savoir le type d'exploitation et les enjeux respectifs au plan de la macro-économie, ne sont pas thématisées dans le Projet qui se borne à l'exploitation du prunus africanus et escamote le problème de la coupe de bois par des agents extérieurs. Ce dernier problème est, bien entendu, enchâssé dans la politique plus générale de l'exploitation des ressources naturelles dont le charbon de bois est un exemple typique dans plusieurs pays. Les licences d'exploitation sont octroyées dans la capitale sur la base de relations de clientélisme et de répartition occultée de profits parallèles, si bien qu'il devient impossible d'appliquer les règlements officiels de gestion à long terme.

Le but officiel du Projet Mount Cameroon est d'établir une planification pour la gestion des ressources forestières par la communauté. A cette fin, le Projet coopère, d'une part, avec les chef traditionnels et, de l'autre, avec les "groupements d'initiative commune" (common initiative groups). La conséquence de l'approche analytique et des démarches qui en découlent, est que la structuration par le genre et par les relations trans-locales du système d'utilisation des ressources naturelles n'est pas prise en compte. Par contre, une image de communauté villageoise est construite, et les femmes sont vues comme un des "groupes d'utilisateurs" marginalisés et sont accusées d'utilisation néfaste des ressources naturelles. Les rapports de genre, la complexité de l'utilisation des ressources, ne sont pas pris en compte. Un discours de paupérisme et de vulnérabilité des femmes est produit et ce discours exclut les femmes comme acteurs économiques : elles sont décrites comme les exploitantes destructrices des ressources naturelles, leur pauvreté étant l'explication de cette conduite. Il ne m'est pas apparu clairement qu'elles étaient aussi considérées comme des usagers illégaux pratiquant des cultures agricoles dans les forêts d'État. Si cela est le cas, il serait aussi très difficile d'améliorer leurs pratiques et leurs technologies, puisqu'elles se seraient mises hors la loi.

L'appréhension de la situation dépend du contenu du concept de communauté locale utilisé. Comment les intérêts et les logiques d'exploitation des femmes, comment les modes d'exploitation coopérative et les rôles complémentaires de genre y sont-ils représentés? En général, les formes parallèles habituelles de représentation des femmes sont rapidement éliminées. La décentralisation accentue cette tendance, tandis que le secteur associatif prévoit certains mécanismes de représentation. L'accès des femmes passe en général par des "big men" modernes ou traditionnels, par leur lignage, leur mari ou encore leur famille d'origine. Les analyses des développeurs ignorent ces aspects relationnels et font passer l'accès aux ressources naturelles des femmes, du niveau de la gestion communautaire, au niveau individuel.

Dans la région du Projet Mount Cameroon, la collecte du bois de chauffage commence à poser un problème, ainsi que l'utilisation de la forêt avoisinante par les femmes, les forêts plus éloignées étant regardées comme domaine des hommes. Jusqu'à l'arrivée du projet, les femmes ramenaient ce bois de chauffage au retour de leur champs de subsistance (chop farms). La privatisation dans le cadre d'une culture "permanente" a rendu leur usage illégal. La logique sous-jacente de l'approche de foresterie sociale et de sa gestion par la communauté implique, an effet, une valorisation économique et un 
calcul individuels de la production : ceci tend à exclure les femmes et par conséquent, à déstructure les modes sexuellement complémentaires de gestion. Le projet incite les femmes à planter des arbres (prunus africanus) dans les champs permanents qui sont clairement la propriété des hommes. De plus, les petits arbres fournis par les pépinières de l'État sont très chers et, de l'avis général, il semble que les femmes devraient les payer elles mêmes.

Une structure néo-traditionelle est ainsi utilisée pour un type moderne de régulation, tout en excluant les femmes de la prise de décision et de la participation de nouvelles opportunités économiques, en dépit de l'utilisation d'une rhétorique de groupement de femmes. J'ai assisté, dans la zone couverte par le Projet, à l'installation du Member Village Traditional Council (Conseil traditionnel des villages membres). Comme dans le temps, il n'y avait pas de membres femmes, sinon deux femmes âgées ayant des fonctions rituelles et une femme membre du parti politique au pouvoir et représentant la promotion féminine. Ainsi, des rapports de genres soi-disant traditionnels ou culturels sont perpétués dans de nouvelles structures sociales et déterminent l'accès à des nouvelles opportunités économiques, sans que se produise un changement social où un ordre de genre serait renégocié. Tout ceci se produit avec l'aide de la Coopération extérieure, qui se plaint, par ailleurs, de la très faible participation des femmes dans les réunions du Projet. Il apparaît clairement que le concept de "communauté" n'inclut pas automatiquement les femmes et que, dans le cas présent, aucun nouvel interface de négociation n'est produit, ni à un niveau supérieur, ni à la base.

Dans l'approche la plus souvent suivie, et illustrée ci-dessus par le cas camerounais, les groupements d'hommes ont un caractère formel, tandis que les groupements de femmes ont un caractère informel. Ailleurs, comme notamment au Sénégal, les hommes seront intégrés dans des Groupements à intérêt économique (GIE) et les femmes dans des groupements de promotion féminine. L'approche des ONG locales intégrées dans le cadre de l'approche participative est extrêmement patriarcale et la quasi totalité des financements extérieurs (par exemple FNUAP) transitent par ces ONG. Partout, on observe comme une quasi fatalité que les petits systèmes locaux de crédits sont considérés comme idéaux pour les femmes, en général pour "le petit commerce", tandis que celles-ci sont exclues des activités incluant de nouveaux modes d'accès aux ressources naturelles et une augmentation de la productivité agricole. Même dans des champs où les femmes étaient porteuses de savoir et de compétence, comme c'était notamment le cas au sud-ouest du Cameroun pour la transformation de miel sauvage, ou au Sénégal dans le cas des rizières des marigots, les projets n'approchent pas les réalités sous un angle relationnel. Et pourtant, la contribution des femmes, et de plus en plus, celle des jeunes filles, devient de plus en plus nécessaire, en raison des migrations masculines.

Les ONG sont, en fait, des acteurs structurant les relations villes-campagnes, bien que de manières assez différenciées. Souvent, les ONG des villes, dirigées par des hommes, perçoivent leur justification dans l'aide à apporter aux femmes pauvres dans les villages : celles-ci sont infantilisées et étiquetées comme ignorantes. De l'autre côté, on voit des femmes urbaines avoir leur propre ONG où l'on retrouve les épouses de président et autres hommes de pouvoir : leurs buts sont, certes, politiques, mais elles ont aussi pour fonction de canaliser les fonds extérieurs en s'ajustant à l'idéologie développementaliste basée sur le passage obligé par les ONG. 

cas du cas du projet sénégalais, tendent à ne pas prendre en compte les relations entre les différents acteurs, leurs modes de coopération et d'interaction, ce qui serait nécessaire pour renforcer la cohésion dans l'action non seulement à l'intérieur d'une entité donnée, mais surtout au delà de celle-ci. Faute d'une approche dynamique et interactive, la communauté risque d'être conçue, soit comme une entité harmonieuse, soit comme un groupement de porteurs d'intérêts, mais, en tout cas, comme un système clos. Les acteurs à la base sont catégorisés selon les clichés d'une dichotomie ville-campagne et/ou d'une opposition d'intérêts hommes-femmes. On en vient à parler, tout simplement, de "villageois" et de "femmes". De façon implicite, le village est donc vu, soit comme une unité sans différenciation sociale, alors que les inégalités se renforcent, en fait, du fait des nouvelles différences d'accès aux ressources extérieures. Il n'est pas plus pertinent de parler de "ménages", si ceux-ci ne sont pas différenciés, de même que doivent l'être les catégories d'âge, les activités économiques, etc.

terme "terroir" utilise présuppose une "communauté ayant une forme d'organisation". Pour la gestion du terroir on parle de l"'affectation des terres à certains usagers" et de "l'affectation d'une fonction". Par contre, la pluralité, la différenciation, la diversité ne sont pas envisagées, pas plus que ne le sont les effets synergiques, les négociations, les intersections. Surtout, la question de l'accès à de nouvelles opportunités socio-économiques (constructions de protection, coopération entre l'élevage et la 
production de bois de chauffage, etc.) sur la base des ressources naturelles n'est ni analysée ni négociée.

Or autant les conflits que les relations d'accès aux ressources importantes dépassent le local et suivent des relations spécifiques entre les locaux et les externes. On le voit, par exemple dans les modèles de gestion et de médiation des conflits entre pasteurs et agriculteurs qui sont au cœur de l'interaction État, économie et société civile (pour les Peuls au Mali, de Bruijn 2000).

Le concept de "communauté" est en principe très bien - c'est-à-dire pragmatiquement défini par le Projet au Sénégal, comme un "cadre de concertation et forum de négociation", dans lequel il est possible de discuter et de négocier, au lieu de concevoir des systèmes différents. Ce qui devient important et qui, jusqu'à présent, n'est pas pris en compte dans la décentralisation, c'est la question des différents niveaux, des interactions entre ces niveaux et des différents responsabilités et règlements potentiellement contradictoires, dans le cas sénégalais, entre les Cellules d'Animation et de Concertation (CAC) et le code forestier, par exemple. Selon le Projet, qui a introduit les CAC regroupant quelques villages suivant des critères écologiques, les limites de la participation sont au niveau de la Communauté rurale. Ceci correspond, pensons-nous, à une approche populiste, alors que, dans la réalité, les interactions et les relations, les négociations avec les niveaux supérieurs doivent toujours être présentes pour accroître ce que nous appelons cohérence verticale dans la Coopération. Par exemple, si l'on parle de "partenariat" avec les Services de l'État, de "contrats de contribution financière des $C R "$, il s'agit déjà d'efforts d'institutionnalisation appropriés, étant donné la crainte des représentants du Projet de ne pas voir les CAC et d'autres conventions reconnues comme légitimes par les échelons supérieurs. Bien sûr, il faudra s'occuper de la question de la compétence technique des CAC, qui certainement, comme cela s'est déjà avéré, n'est souvent plus adaptée. Le recours du Projet à des services professionnels, par exemple pour la construction d'ouvrages de protection des ressources naturelles, inquiète les membres des organisations paysannes. Ceci montre bien la nécessité de négocier l'enchâssement du Projet dans les structures des services publiques existants, tout en tenant compte de l'opportunité qui se présente ainsi de renforcer la société civile dans son interaction avec l'état autoritaire.

On peut donc presque parler de compétition entre État et société rurale au Sénégal concernant les services à rendre sur la base des types de savoir et des approches modernistes ou "appropriées-rurales". C'est le cas pour la question des puits villageois (construction et, surtout, entretien) et aussi pour les nouvelles formes de gestion monétaire de l'économie rurale. Les Caisses Populaires Sénégalaises gagnent en envergure ces dernières années, des professionnels ruraux, provenant de zones rurales travaillent dans des petites villes rurales. Cette approche semble concurrencer les formes antérieures de systèmes épargne/crédit à travers lesquels le mouvement paysan a essayé, sans grand succès, de faire face au malaise paysan. Entre temps, des leaders du mouvement paysan, et des professionnels issus de ce milieu, ont mis au point de nouveaux modèles, en collaboration avec des spécialistes formés dans des institutions trans-nationales. Ici, l'objectif est d'une autonomie quasi-totale en vue d'une gestion différente de l'argent.

Sur le plan des relations de savoir, il est très frappant de constater qu'aussi bien les conseillères rurales que les responsables femmes d'organisations paysannes 
considèrent comme le monopole des nouveaux savoirs et des informations par les hommes est l'un de leurs plus grands problèmes. En ce qui concerne les ressources naturelles, les femmes ont, jusqu'à présent, été uniquement sensibilisées à la construction et à l'utilisation de foyers améliorés (bann ak suuf). Les réseaux spécialisés sur des thèmes spécifiques (certaines ressources naturelles, épargne et crédit, etc.), et visant une réelle extension des connaissances, font défaut. Ces réseaux devraient inclure des représentants des organisations paysannes et de femmes, des responsables locaux, des représentants des $\mathrm{CR}$ et d'autres porteurs de savoir dans la zone, telles que les animateurs et les promoteurs du mouvement paysan, souvent formés depuis un certain temps et au fait des traditions locales, ce qui semble oublié. Faute de s'appuyer sur ces réseaux, les $\mathrm{CR}$ risquent, par les nouvelles pratiques de distribution de terres, d'établir des nouvelles relations de clientélisme, de mercantiliser l'accès à la terre et aux ressources naturelles et ainsi, à exclure les femmes.

Conclusion

Les re-configurations des relations sociales et économiques ont été analysés tout en élaborant sur la structuration des champs d'activités par genre. Le concept d'enchâssement, redécouvert dans la sociologie économique récente, le concept d'une trans-localité constitutive des processus de mondialisation, le recours aux analyses empiriques et débats théoriques de la recherche sur le genre, ont montré ici leur efficacité méthodologique.

L'intention, à première vue peut-être très ambitieuse, d'intégrer une perspective genre aux nouveaux débats théoriques des sciences sociales concernant les phénomènes de la mondialisation pour augmenter leur pouvoir explicatif, a, semble-t-il, été fructueuse. La démarche a permis d'élaborer des concepts de théorie d'envergure moyenne (middle range theory) sur des donnée empiriques. Tant l'enchâssement de l'économie que la trans-localité peuvent être concrétisés et s'avèrent utiles pour l'étude de l'économie locale et trans-locale, tout en montrant que des "recompositions sociales, relations à distance et logiques des acteurs" - pour reprendre le titre du groupe de travail à la conférence APAD en 2000 où cet article a été présenté - sont souvent structurées par le genre.

70 Les méthodologies proposées, fondées sur les interfaces et les modes d'accumulation, semblent avoir un haut degré de pouvoir explicatif, mettant en avant le caractère relationnel, systémique, et la dynamique du champ économique conçu comme structuré par le social. Les exemples et les études de cas en Afrique de l'Ouest montrent que les transformations en cours ne sont pas comprises dans leurs réalités sociales et que, de plus, les approches genre appliquées dans la Coopération sont extrêmement étroites. Avec le changement de paradigme on peut, en effet, dépasser les dualismes toujours existants et concevoir d'autres perspectives tout en se concentrant sur les processus en cours, telle que l'informalisation ou l'envergure effective des activités considérées comme locales.

71 En analysant l'économie féminine sous le paradigme de l'enchâssement, il a été possible de capter cette complexité dans le contexte relationnel et trans-local des différents champs d'activités, l'organisation des liens sociaux qui unissent entre ville et campagne, par exemple la coopération entre épouses ou entre différents ménages. La monétarisation y joue un rôle important en ce qui concerne l'accès aux ressources foncières, financières et autres. La constitution des bases d'existence (livelihood) a été 
mise en avant à travers les relations entre villes et campagnes, production de subsistance et marché, et la migration comme guidant les relations correspondantes.

Il devient évident que souvent les femmes et leur activités se voient assimilées au local et au rural, le tout dans un processus de pensée extrêmement réducteur. Si, par contre, on essaie de concevoir les relations urbaines - rurales comme structurées par le genre, il devient possible de dépasser les dualismes et d'ouvrir de nouvelles perspectives dans l'étude du commerce à distance ou de la signification économique des nouveaux efforts de décentralisation ou de démocratisation.

Le secteur associatif s'est avéré un acteur de l'espace trans-local au sein duquel les relations de genre jouent un rôle important. Les différences marquées par le genre apparaissent en ce que certaines femmes agissent en effet comme courtiers de développement, mais que les questions de volontariat ou de professionnalisation et d'accès au savoir se posent en général pour les hommes. Dans ce contexte, la sécurité alimentaire apparaît comme un champ à la fois économique et politique et des modes d'activités économiques transformatrices qui pourraient être développées dans ces espaces par des acteurs de la société civile peuvent être étudiées.

Lorsque l'on considère les relations entre État urbain et société rurale dans le cadre de la décentralisation, et que l'on aborde ces relations sous l'angle des modes autoritaires de gestion de l'économie rurale, on peut conclure que les dispositifs de développement risquent de perpétuer la perception dichotomique des villes et des campagnes, oubliant le contexte social et politique auquel les rapports de genre sont transversaux.

Les deux exemples étudiés, celui de la Coopération économiques concernant l'introduction du concept de la foresterie social au Cameroun et celui de l'introduction d'un organisme de coordination par auto-promotion des ressources naturelles au Sénégal, montrent que, pour résoudre les problèmes opérationnels d'action économique et d'action collective, les relations sociales et leur structuration par le genre doivent être analysés. Une approche théorique plus complexe doit être adoptée.

\section{BIBLIOGRAPHIE}

Amponsem G., 1996, Global trading and business networks among Ghanaians : an interface of the local and the global, Université de Bielefeld, Faculté de Sociologie (thèse de doctorat non publ.).

Appadurai A., 1991, "Global ethnoscapes : notes and queries for a transnational anthropology", in Fox, Richard G. (éd.), Recapturing anthropology. Working in the present, Santa Fe N.M., School of American Research Pro : 191-210.

Appadurai A., 1998, Modernity at large. Cultural dimensions of globalization, Minneapolis, Univ. of Minneapolis Pr.

Arce A., Long N. (éds), 2000, Anthropology, development and modernities. Exploring discourses, counter-tendencies and violence, London, New York, Routledge. 
Bazenguissa-Ganga R., 2000, "Les commerçants "clandestins" congolais à Paris", in Engel, U., A. Jones, R. Kappel (éd.), VAD German African Studies Association $17^{\text {th }}$ Biennial Conference Leipzig 30.3. 1.4. (CD).

Becher C., 1996, "'Das Land gehort zu den Miinnern" : Landzugang von Frauen im Norden Ghanas im Rahmen dynamischer Transformationsprozess", Univ. Bielefeld, Fac. de Sociologie ( thèse de diplôme non publ.).

Bierschenk T., Chauveau J.-P., Olivier de Sardan J.-P., 2000, "Introduction : Les courtiers entre développement et État", in idem (éds), Courtiers en développement. Les villages africains en quête de projets, Paris, APAD, Karthala : 5-42.

Blundo G., 1994, "Le conflit dans l'Entente. Coopération et compétition dans les associations paysannes du bassin arachidier sénégalais", in J.-P. Jacob, Ph. Lavigne Delville (éd.) : 99-120.

Blundo G., 1995, "Les courtiers du développement en milieu rural sénégalais", Cahiers d'Etudes Africaines, La démocratie déclinée, 24 -1 (137) : 73-99.

Bruijn M. de, 2000, "Rapports interethniques et identité : l'exemple des pasteurs peuls et des cultivateurs hummbeeBe au Mali central", in Diallo, Youssouf, Günther Schlee (éds), L'ethnicité peule dans des contextes nouveaux, Paris, Karthala : 15-36.

Desai B., 1999, Knowledge, Gender, and institutions in Agriculture : an example from research in Africa, Université de Bielefeld, Fac. de Sociologie (thèse de diplôme non pub !).

Diop A., 1995, Femme et pouvoir politique : quelles perspectives pour la régionalisation, Journée internationale de la Femme 8 Mars, Dakar, Fondation Friedrich Ebert.

Dwyer D., Judith B. (éds), 1988, A home divided. Women and income in the Third World, Stanford, Calif., Stanf. Univ. Pr.

Elson D., 1995, "Gender awareness in modeling structural adjustment", in Cagatay, Nilüfer, Diane Elson, Caren Grown (éds) Gender, adjustment and macroeconomics, Special issue in World Development 23 (11) : 1851-1868.

Elwert G., 1987, "Ausdehnung der Kauflichkeit und Einbettung der Wirtschaft - Markt und Moralokonomie", Kolner Zeitschriftfür Soziologie und Sozialpsychologie, nº spécial 28 : 300-321.

Engel U., Jones A., Kappel R. (éds), 2000, VAD German African Studies Association 17th Biennial Conference Leipzig 30.3. - 1.4.2000 (CD).

Fall A.S., Serigne M., 1999, Les interactions rurales et urbaines : circulation et mobilisation des ressources, Appel à contribution pour Séminaire scientifique de l' APAD, Sénégal, 26-29.1.2000 (Ms.).

Fisiy C.F., Geschiere P., 1993, "Sorcellerie et accumulation, variations régionales", in : P. Geschiere, P. Konings (éds) : 99-130.

Geschiere P., 1997, The modernity of witchcraft, Politics and the occult in postcolonial Africa, Charlotteville, London, Univ. Pr. of Virginia.

Geschiere P., Konings P., 1993, "Introduction : Alternative modes of accumulation and their regional variations", in idem (dir.), Itinéraires d'accumulation au Cameroun, Paris, Leiden, Karthala : 9-31.

Granovetter M., 1985, "Economic action and social structure : the problem of embeddedness", American Journal of Sociology, $3: 481-510$. 
Granovetter M. 1992. "The sociological and economic approaches to labor market analysis : a social structural view", in idem, Richard Swedberg (éd.), The sociology of economic life, Boulder : Westview Pr : 233-264.

Guyer J., 1988, "Dynamic approaches to domestic budgeting : cases and methods from Africa", in Dwyer D., L. Bruce (éds) : 155-172.

Hyden G., 1980, Beyond Ujamaa in Tanzania. Underdevelopment and an uncaptured peasantry, London etc., Heinemann.

Jacob J.-P., Philippe Lavigne Delville (dir.), 1994, Les associations paysannes en Afrique, Organisation et dynamiques, Paris, Karthala.

Joekes S., Naila K. (éds), 1991, "Researching the household : methodological and empirical issues", IDS, Bulletin 22 (1).

Laaser M., 2001, Business women in Nairobi / Kenya, Université de Bielefeld, Centre de Sociologie en Développement (Ms.).

Lachenmann G., 1992, Die Gefährdung sozialer Sicherung in der Krise und Perspektiven neuer Strategien der Selbstorganisation : Fall Kamerun, Berlin : Deutsches Institut für Entwicklungspolitik (Institut Allemand de Développement).

Lachenmann G., 1994, "Civil society and social movements in Africa", in Jacob J.-P., Ph. Lavigne Delville (dir.) : 61-95.

Lachenmann G. et al., 1995, Lehrforschung Transformationsprozesse in Sénégal, Version réduite en fr. : Rapport d'étude de terrain. Processus de transformation en Afrique de l'Ouest, Université de Bielefeld, Centre de Recherche en Sociologie de Développement.

Lachenmann G., 1996a, "Transformationsprozesse in Westafrika - Widersprüche und Chance für Frauenpolitik und Wandel der Geschlechterverhaltnisse", Afrika. Asien. Lateinamerika 24 : 231-251.

Lachenmann G., 1996b, Stiirkung von Genderaspekten im Rahmen von Vorhaben der deutschen 12 in Kamerun, Renforcer les aspects genre dans le cadre de la coopération germano-camerounaise, "Why gender matters", Expertise pour GTZ (Ms.).

Lachenmann G., 1997, "Future perspectives of rural women's projects - intervention, interaction or empowerment ?", in Altmann, Uta, Parto Teherani-Kronner (éds), What have women 's projects accomplished so far? Proceedings of the 2nd. international conference "Women in Rural Development, Humboldt University, Berlin, June 28-30,1996 : 31-52.

Lachenmann G., 1998a, Genre, pauvreté et développement. Avec exemples du Bénin/Afrique de l'Ouest, Working Paper 307, Université de Bielefeld, Centre de Recherche en Sociologie du Développement (http://www.uni-bielefeld.de/sdrc/homesdrc).

Lachenmann G., 1998b, "ONG : force sociale dans les transformations en cours et relations de genre", in Deler, J.P., Y.-A. Fauré, A. Piveteau, P.-J.Roca (éds), ONG et développement, Société, économie, politique, Paris, Karthala : 107-114.

Lachenmann G., 1999a, Engendering embeddedness of economy in society and culture, Working Paper 323, Université de Bielefeld, Sociology of Development Research Centre (http://www.unibielefeld.de/sdrc/homesdrc).

Lachenmann G. et al., 1999b, Lehrforschung Zivilgesellschaft in Senegal : der Beitrag der Bauernorganisationen, version réduite en fr. : Rapport d'étude de terrain. Société civile au Sénégal : la contribution des organisations paysannes. Projet de recherche en Afrique de l'Ouest, Université de Bielefeld, Centre de Recherche en Sociologie de Développement. 
Lambert A., 1993, "Les commerçantes maliennes du chemin de fer Dakar-Bamako", in Emmanuel Grégoire, Pascal Labazée (dir.), Grands commerçants d'Afrique de l'Ouest. Logiques et pratiques d'un groupe d'hommes d'affaires contemporains, Paris, Karthala, Orstom : 37-70.

Long N., 1992, "Introduction ; From paradigm lost to paradigm regained ? The case for an actor-oriented sociology of development ; Conclusion", in idem., A. Long (éd.) : 3-15 ; 268-277 ; $16-46$.

Long N., 1996, "Globalization and localization : new challenges to rural research", in Moore, Henrietta (éd.), The future of anthropological knowledge, London, Routledge : 37-59.

Long N., 2000, "Exploring local/global transformations : a view from anthropology", in Arce, A., idem : 184-201.

Long N., Long A. (éds), 1992, Battlefields of knowledge. The interlocking of theory and practice in social research and development, London, Routledge.

Mbembe A., 1988, "État, violence et accumulation. Leçons d'Afrique noire", in Foi et développement, 164/165:1-8.

Müller C. 1999. Geschlechtsspezifisches Wissen in Ghana, Vortrag bei der Sektion Entwicklungssoziologie/Sozialanthropologie, Hohenheim.

Ndione E.S., 1992, Le don et le recours. Ressorts de l'économie urbaine, Collection Recherches Populaires, $\mathrm{n}^{\circ}$ 151-153, Dakar, Enda-Editions.

Nageeb S., 2000, Space Practice in Sudan : Reconsidering Women's Seclusion, Université de Bielefeld, Fac. de Sociologie (thèse de doctorat non publ.).

N'Sangou A., 1985, "La contribution des buy'em sell'em au développement", in Barbier J.-C. (s.l.d.d.) : 385-392.

Nzomo M., 1995, "Women and democratization struggles in Africa : what relevance to post-modemist discourse ? ", in Marchand Marianne H., Jane L. Parpart (éds), Feminism, postmodernism, development, London, Routledge : 131-141.

Padmanahban M.A., 2000, Gender relations and agricultural innovations in Northern Ghana, Université de Gottingen, Fac. des Sciences Agricoles (thèse de doctorat non publ.).

Peleikis A., 1998, Lebanese in Motion. The making of a gendered, globalized village, Université de Bielefeld, Fac. de Sociologie (thèse de doctorat non publ.).

Polanyi K., 1987, The great transformation. Politische und okonomische Ursprünge von Gesellschaften und Wirtschafssystemen, Frankfurt a.M, Suhrkamp.

Santen José C.M. van, 1998, "Islam, Gender and urbanisation among the Mafa of north Cameroon : the differing commitment to, home "among Muslims" and "non-Muslims"", Africa. 68 (3) : 403-424.

Schneider G., 1999, Women's economy between subsistence and market production in Harare, Zimbabwe, Working Paper 315, Université de Bielefeld, Centre de Recherche en Sociologie de Développement (http://www.uni-bielefeld.de/sdrc/homesdrc).

Schneider G., 2000, "Local and translocal economy. Embeddedness of female economy in urban Zimbabwe.", in Engel U., A. Jones, R. Kappel (éds), VAD German African Studies Association $17^{\text {th }}$ Biennial Conference Leipzig 30.3. - 1.4. (CD).

Schneider G., 2000, Frauenokonomie zwischen Subsistenzwirtschaft und Markt in Harare-Zimbabwe, Université de Bielefeld, Fac. de Sociologie (thèse de doctorat non publ.). 
Schultz U., 1996, Nomadenfrauen in der Stadt. Die Überlebensokonomie der Turkanafrauen in LodwarINordkenia, Berlin, Reimer.

Sen A., 1981, Poverty and famines. An essay on entitlement and deprivation. New York, Oxford Univ. Press.

Waibel G., 1998, Sesshaftwerdung und sozialer Wandel bei den Tuareg Zinders (Niger), Hamburg, Institut für Afrika-Kunde.

Wanzala W., 2000a, "Embeddedness of Female Economy in Urban Namibia", in Engel U., A. Jones, R. Kappel (éds), VAD German African Studies Association l7'h Biennial Conference Leipzig 30.3. - 1.4. (CD).

Wanzala W., 2000b, Women in the Informal Sector of Urban Namibia, Université de Bielefeld, Fac. de Sociologie (thèse de doctorat non publ.).

\section{NOTES}

1.Une version précédente de ce texte a été présentée au colloque APAD tenu à saint-Loius (Sénégal) du 26 au 28 janvier 2000. Ce colloque portait sur "Les interactions rurales et urbaines : circulation et mobilisation des ressources", Le texte a été présenté à l'Atelier 5 : "Recompositions sociales, relations à distance et logiques des acteurs". 2.Ex. Ghana : Les Queen Mothers (étude de Christine Müller) essaient de s'inclure dans l'effort de centrer l'amorce principale de la structure 'traditionelle' dans les structures au niveau national (House of Chiefs, resp. Association of Queen Mothers). ce qui peut être considéré que le local constitué dans le "traditionnel" devient mono-genre.

\section{AUTEUR}

\section{GUDRUN LACHENMANN}

Centre de Recherche en Sociologie du Développement, Université de Bielefeld, Allemagne. 